\title{
New Analysis Method for Wireless Power Transfer System with Multiple $n$ Resonators
}

\author{
Ju-Hui Kim $\cdot$ Byung-Chul Park $\cdot$ Jeong-Hae Lee
}

\begin{abstract}
This paper presents a new method for analyzing the maximum efficiency of a wireless power transfer (WPT) system with multiple $n$ resonators. The method is based on ABCD matrices and allows transformation of the WPT system with multiple $n$ resonators into a single two-port network system. The general maximum efficiency equation of a WPT system with multiple $n$ resonators is derived using the ABCD matrix. Use of this equation allows placement of the relay resonators for maximum efficiency even though they are asymmetrical. The general maximum efficiency equation and the method of the optimum placement are verified by a full wave simulation. The results show that the method is useful for the analysis of a WPT system with relay resonators.
\end{abstract}

Key Words: ABCD Matrix, Optimum Placement, Relay Resonators, Two-Port Network Theory, Wireless Power Transfer.

\section{Introduction}

Many researchers are currently investigating magnetically coupled wireless power transfer (WPT) systems. A conventional WPT system consists of two main resonators for the transmitter and receiver, which operate at the same resonance frequency [1-3]. This system, however, has a limitation on the distance between transmitter and receiver for achieving high WPT efficiency. This limitation can be overcome by using some resonators as relays between the two main resonators [4]. The WPT system is then composed of multiple $n$ resonators, which can achieve a higher WPT efficiency than a conventional WPT system can at the same distance. This system is well analyzed in [5] with respect to the general circuit model and the maximum efficiency with a matched load. However, the general maximum efficiency equation of a WPT system with multiple $n$ resonators described in [5] becomes complicated when analyzing the type of system normally utilized for practical applications.

This paper proposes a new analysis method for the WPT system with multiple $n$ resonators. The method simplifies the entire WPT system into a single two port network expressed by an ABCD matrix. The general maximum efficiency equation of the WPT system with multiple $n$ resonators can then be obtained with elements of the ABCD matrix. This equation provides a much more convenient way to obtain the maximum efficiency and the optimum placement of the asymmetrical relays for the best utilization. The general maximum efficiency equation and the method of the optimum placement of the asymmetrical relays are verified by a full wave simulation of ANSYS HFSS (high frequency structural simulator).

\section{New Analysis Method Using the ABCD Matrix}

The schematic diagram of a WPT system with multiple $n$ resonators is shown in Fig. 1(a). The non-adjacent coupling coefficient is ignored since it is very small in a practical WPT system with the relays. Thus, the WPT system with multiple $n$ resonators can be considered as $n-1$ parts of two-port networks connected in series, as shown in Fig. 1(b). Two-port networks have three types of connections: transmitter-relay, relay-relay, and relay-receiver. The system in Fig. 1(b) can then be represented as the single two-port network shown in Fig. 1(c) by the matrix multiplication of $\mathrm{ABCD}$ matrices. The maximum efficiency equation in [1] can be adapted for

Manuscript received May 23, 2013 ; Revised June 25, 2013 ; Accepted June 27, 2013. (ID No. 20130523-026J)

Department of Electronic Information and Communication Engineering, Hongik University, Seoul, Korea.

${ }^{*}$ Corresponding Author : Jeong-Hae Lee (e-mail : jeonglee@hongik.ac.kr)

This is an Open-Access article distributed under the terms of the Creative Commons Attribution Non-Commercial License (http://creativecommons.org/licenses/ by-nc/3.0) which permits unrestricted non-commercial use, distribution, and reproduction in any medium, provided the original work is properly cited. 


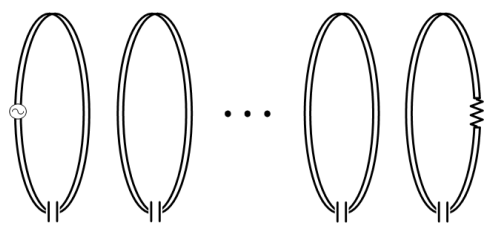

(a)

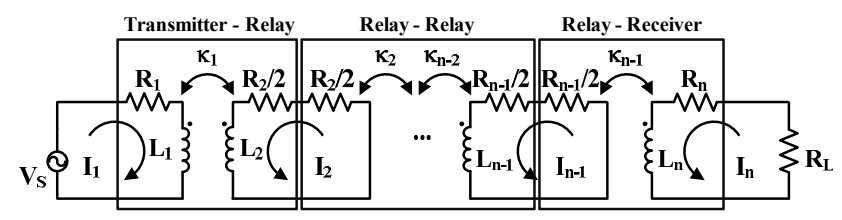

(b)

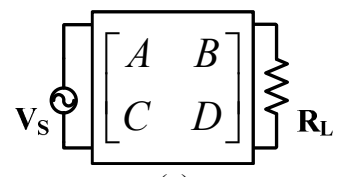

(c)

Fig. 1. (a) Wireless power transfer (WPT) system with $n$ resonators, (b) the equivalent circuit model of a WPT system with $n$ resonators at the resonance frequency $\left(f=f_{0}\right)$, and (c) the equivalent two-port network of a WPT system with $\mathrm{n}$ resonators.

this single equivalent two-port network system because the WPT system with two resonators is also a single two-port network. $R_{L}$ at the end of the circuits in Fig. 1(b) and (c) is a matched load required to obtain the maximum efficiency of the WPT system. The matched load $R_{L}$ can be connected to the receiver either directly or indirectly by inductive coupling with the feeding loop. When the load impedance $Z_{L}$ is connected through the feeding loop, $R_{L}$ can be expressed with the elements of a feeding loop as:

$$
R_{L}=\frac{\left(\omega_{0} \kappa_{s} \sqrt{L L_{f}}\right)^{2}}{Z_{L}+j \omega_{0} L_{f}}
$$

where $\kappa_{s}$ is a coupling coefficient between a feeding loop and the receiver, $L$ is the inductance of receiver, and $L_{f}$ is the inductance of a feeding loop.

In order to obtain $\mathrm{ABCD}$ matrices for three types of two-port networks shown in Fig. 1(b), the circuit equations of a WPT system are needed. When the system operates at the resonance frequency $\left(f=f_{0}\right)$, the circuit equations derived from Fig. 1(b) are the following equations:

$$
\begin{aligned}
& R_{1} I_{1}+j \omega_{0} \kappa_{1} \sqrt{L_{1} L_{2}} I_{2}=V_{s} \\
& j \omega_{0} \kappa_{i-1} \sqrt{L_{i-1} L_{i}} I_{i-1}+R_{i} I_{i} \\
& \quad+j \omega_{0} \kappa_{i} \sqrt{L_{i} L_{i+1}} I_{i+1}=0(i=2,3, \cdots, n-1)
\end{aligned}
$$

Table 1. The $Z$ matrix and $A B C D$ matrix of each divided two-port network of a wireless power transfer system

\begin{tabular}{ccc}
\hline & Z matrix & ABCD matrix \\
\hline $\begin{array}{c}\text { Transmitter } \\
\text { Relay }\end{array}$ & $\left(\begin{array}{cc}R_{1} & j C_{1} \\
j C_{1} & \frac{R_{2}}{2}\end{array}\right)$ & $\left(\begin{array}{cc}\frac{R_{1}}{j C_{1}} & \frac{R_{1} R_{2}+2 C_{1}^{2}}{j 2 C_{1}} \\
\frac{1}{j C_{1}} & \frac{R_{2}}{j 2 C_{1}}\end{array}\right)$ \\
$\begin{array}{c}\text { Relay } \\
\text { Relay }\end{array}$ & $\left(\begin{array}{cc}\frac{R_{i-1}}{2} & j C_{i-1} \\
j C_{i-1} & \frac{R_{i}}{2}\end{array}\right)$ & $\left(\begin{array}{cc}\frac{R_{i-1}}{j 2 C_{i-1}} & \frac{R_{i-1} R_{i}+4 C_{i-1}^{2}}{j 4 C_{i-1}} \\
\frac{1}{j C_{i-1}} & \frac{R_{i}}{j 2 C_{i-1}}\end{array}\right)$ \\
Relay & $\left(\begin{array}{ccc}\frac{R_{n-1}}{j 2 C_{n-1}} & \frac{R_{n-1} R_{n}+4 C_{n-1}{ }^{2}}{j 4 C_{n-1}} \\
\frac{1}{j C_{n-1}} & \frac{R_{n}}{j 2 C_{n-1}}\end{array}\right)\left(\begin{array}{cc}\frac{R_{n-1}}{j 2 C_{n-1}} & \frac{R_{n-1} R_{n}+2 C_{n-1}^{2}}{j 2 C_{n-1}} \\
\frac{1}{j C_{n-1}} & \frac{R_{n}}{j C_{n-1}}\end{array}\right)$ \\
\hline
\end{tabular}

$$
j \omega_{0} \kappa_{n-1} \sqrt{L_{n-1} L_{n}} I_{n-1}+\left(R_{n}+R_{L}\right) I_{n}=0
$$

Then, $\mathrm{Z}$ matrices of each two-port network are obtained by (2)-(4), and they can be transformed into ABCD matrices [6] following Table 1, where $C_{i-1}$ is $\omega_{0} \kappa_{i-1} \sqrt{L_{i-1} L_{i}}(i=2,3, \cdots, n)$, and $\kappa_{i-1}$ is the coupling coefficient between $i$-1-th resonator and $i$-th resonator. The single ABCD matrix of the WPT system with multiple $n$ resonators can then be obtained. Finally, the system is equivalent to a WPT system with two resonators.

The maximum efficiency $\left(\eta_{\max }\right.$ ) equation of the WPT system with two resonators can be applied to the WPT system with multiple $n$ resonators:

$$
\eta_{\max }=\left(\frac{U}{1+\sqrt{1+U^{2}}}\right)^{2}
$$

where $U=\kappa_{1} \sqrt{Q_{1} Q_{2}}$ is the figure of merit of a WPT system with two resonators, and $Q_{i}=\omega_{o} L_{i} / R_{i}(i=1,2)$ is the quality factor of each resonator [1]. The figure of merit $U$ can also be written in terms of elements of the $\mathrm{Z}$ matrix and $\mathrm{ABCD}$ matrix:

$$
U=\kappa \sqrt{Q_{1} Q_{2}}=j \sqrt{\frac{Z_{12} Z_{21}}{Z_{11} Z_{22}}}=j \sqrt{\frac{1}{A D}}
$$

where $Z_{i j}(i=1,2$ and $j=1,2)$ is the element of the $Z$ matrix, and $A$ and $D$ are the elements of the ABCD matrix of the WPT system with 2 resonators. The figure of merit $U$ in terms of the elements of ABCD matrix can be applied to a WPT system with multiple $n$ relays using the elements of its single $\mathrm{ABCD}$ matrix and it is re- 
named $U^{\prime}$.

The Eq. (5), $\eta_{\max }$, should also be modified to make a general maximum efficiency equation because Eq. (5) is suitable only for a WPT system with two resonators. The WPT systems with multiple $n$ resonators are classified into two types of systems by the phase difference of the current on the receiving resonator. The phase difference is $\pi / 2$ between the odd and even numbers of resonators. Thus, the modification of phase for power is $j^{2}$, which is multiplied in Eq. (5) when the number of resonators is odd. Finally, the general maximum efficiency equation of a WPT system with multiple $n$ resonators is given by:

$$
\eta_{\max }=(-1)^{n}\left(\frac{U^{\prime}}{1+\sqrt{1+U^{\prime 2}}}\right)^{2}
$$

As an example, the maximum efficiency equations of WPT systems with three and four resonators derived by (6) and (7) are represented as:

$$
\begin{aligned}
& \eta_{\max }=\frac{\frac{U_{1}^{2} U_{2}^{2}}{\left(1+U_{1}^{2}\right)\left(1+U_{2}^{2}\right)}}{\left(1+\sqrt{\left.1-\frac{U_{1}^{2} U_{2}^{2}}{\left(1+U_{1}^{2}\right)\left(1+U_{2}^{2}\right)}\right)^{2}}\right.} \\
& \eta_{\max }=\frac{\frac{U_{1}^{2} U_{2}^{2} U_{3}^{2}}{\left(1+U_{1}^{2}+U_{2}^{2}\right)\left(1+U_{2}^{2}+U_{3}^{2}\right)}}{\left(1+\sqrt{1+\frac{U_{1}^{2} U_{2}^{2} U_{3}^{2}}{\left(1+U_{1}^{2}+U_{2}^{2}\right)\left(1+U_{2}^{2}+U_{3}^{2}\right)}}\right)^{2}}
\end{aligned}
$$

where $U_{i-1}^{2}=\kappa_{i-1}^{2} Q_{i-1} Q_{i}(i=2,3, \cdots, n)$ is the square of figure of merit between $i$ - 1 -th resonator and $i$-th resonator. $Q_{i}=\omega_{0} L_{i} / R_{i}$ is the quality factor of $i$-th resonator. The matched load $R_{L}$ is also expressed with the elements of ABCD matrix.

$$
R_{L}=\sqrt{\frac{B D}{A C}}
$$

The maximum efficiency of a WPT system with multiple $n$ resonators and the matched load $R_{L}$ can then be obtained by the elements of the single equivalent ABCD matrix for the system, (6), (7), and (10). These equations provide a much simpler form than those in [5].

The maximum efficiencies calculated by Eq. (7) are compared with those simulated by ANSYS HFSS, as shown in Fig. 2. The systems operate at $6.78 \mathrm{MHz}$ and have three and four identical resonators, as shown in Fig. 2(a) and (b), respectively. The square resonators [7]

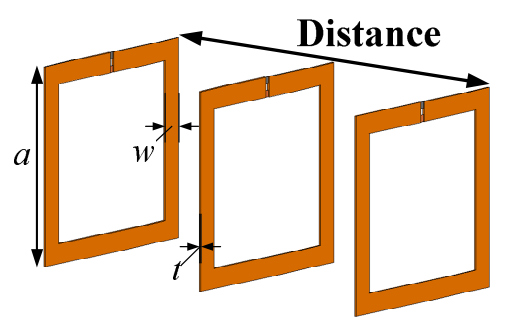

(a)

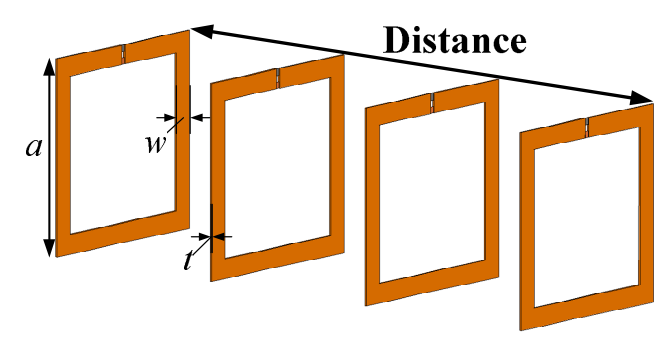

(b)

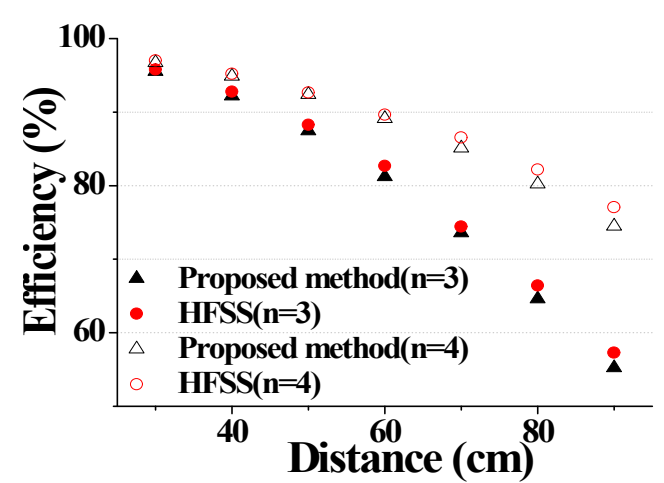

(c)

Fig. 2. (a) Wireless power transfer (WPT) system with three identical resonators, (b) WPT system with four identical resonators, and (c) maximum efficiency of WPT systems with three and four resonators at $6.78 \mathrm{MHz}$. HFSS=high frequency structural simulator.

used for this system have the following dimensions: $a=20 \mathrm{~cm}, w=2 \mathrm{~cm}, t=0.2 \mathrm{~cm}$, and $C=1,498 \mathrm{pF}$, and the material of resonators is assigned as copper. The total distance of both systems is changed from 30 to $90 \mathrm{~cm}$. The resonators in the WPT system are equally spaced and have $Q_{i}$ of 950, and the coupling coefficient $\kappa_{i}$ is found from even and odd mode analysis at each corresponding distance between two adjacent resonators [1]. In Fig. 2(c), the efficiencies obtained by two different methods agree well, indicating the validation of the derived equations. However, small differences appear as the distance increases due to the non-adjacent coupling. As the distance gets progressively longer, the difference between the adjacent and non-adjacent coupling coefficient become smaller and, thus, the non-adjacent coupling coefficient has a much stronger influence on the 
efficiency.

\section{Optimum Placement of Asymmetrical Relays}

The optimum placement of a WPT system with the asymmetrical relay resonators can also be found with (7). When the WPT system has different sizes of resonators or more than 2 relays, the maximum efficiency will be determined not by the equal spacing but by the optimum placement. The optimum placement should be found by observing the maximum efficiencies at the various placements. These can be easily obtained by calculating (7) with respect to different placements. The optimum placement can then be found from the peak value of the calculation from (7).

This method is verified by constructing the WPT system with four resonators shown in Fig. 3(a). The system operates at $6.78 \mathrm{MHz}$ and has four square resonators, as shown in Fig. 3(a). Each resonator has $Q_{1}=Q_{4}$ of 878, $Q_{2}$ of $1,004, Q_{3}$ of 567 , and the adjacent coupling coefficients are changed by the different distances. The square resonators [7], assigned as copper, used for this system have the following dimensions: $a_{1}=a_{4}=20 \mathrm{~cm}$, $a_{2}=30 \mathrm{~cm}, a_{3}=10 \mathrm{~cm} ; w_{1}=w_{2}=w_{4}=2 \mathrm{~cm}, w_{3}=1.5 \mathrm{~cm} ; t=0.2$ $\mathrm{cm}$; and $C_{1}=C_{4}=1,467 \mathrm{pF}, C_{2}=804 \mathrm{pF}, C_{3}=3,860 \mathrm{pF}$. The total distance between the transmitter and receiver is 75

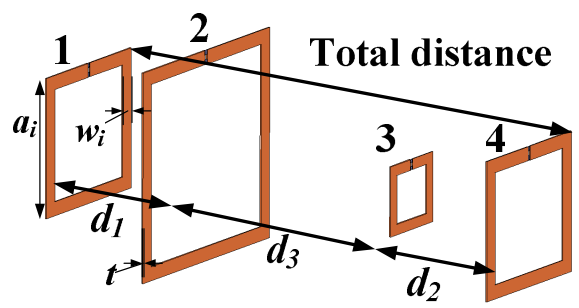

(a)

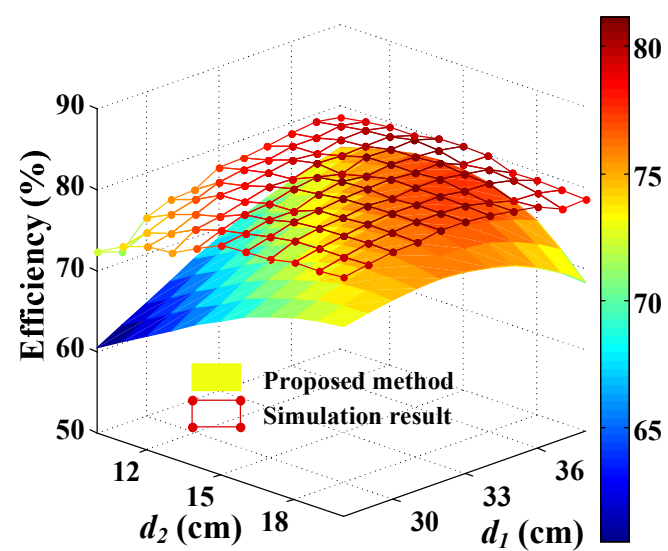

(b)

Fig. 3. (a) Wireless power transfer (WPT) system with two asymmetric relay resonators and (b) maximum efficiency having different size of relay resonators at $6.78 \mathrm{MHz}$ $\mathrm{cm}$. The optimum distance of $d_{1}$ and $d_{2}$ should be determined to obtain the maximum efficiency. The maximum efficiencies calculated using Eq. (7) are compared with those simulated by ANSYS HFSS, as shown in Fig. 3(b), changing $d_{1}$ and $d_{2}$ from 28 to $38 \mathrm{~cm}$ and from 10 to $20 \mathrm{~cm}$, respectively. The results from calculation and simulation are obtained at every $1 \mathrm{~cm}$. The coupling coefficients used for calculated maximum efficiencies are calculated from the field calculation of $H_{Z}$ in [8]. The maximum efficiencies and the optimum placement of the WPT system for the verification can then be found from simulation and calculation. The optimum placement is found at $d_{1}=34 \mathrm{~cm}, d_{2}=16 \mathrm{~cm}$ with the efficiency of $81.17 \%$ from the simulation and at $d_{1}=37 \mathrm{~cm}, d_{2}=15 \mathrm{~cm}$ with the efficiency of $77.3 \%$ from the calculation. The results show that the tendency for variation in both methods agrees well, despite the small difference caused by the non-adjacent coupling. Thus, the maximum efficiency and the optimum placement can be found quite simply using the proposed calculation.

\section{Conclusion}

A simple method to obtain the maximum efficiency of a WPT system with multiple $n$ resonators is presented in this paper. This method is based on ABCD matrices and allows the WPT system with multiple $n$ resonators to be transformed into a single two-port network system. The general maximum efficiency equation of a WPT system with multiple $n$ resonators is derived using the $\mathrm{ABCD}$ matrix and is verified by a full wave simulation. This equation is also applied to a WPT system with asymmetrical relays for placement of the relays for the maximum efficiency. The method of optimum placement is also verified by a full wave simulation. The results indicate that our method can be extended to analyze WPT systems with asymmetrical relay resonators.

This research was funded by the Ministry of Science, ICT \& Future Planning, Korea in the ICT R\& D Program 2013.

\section{References}

[1] A. Kurs, A. Karalis, R. Moffatt, J. D. Joannopoulos, P. Fisher, and M. Soljacic, "Wireless power transfer via strongly coupled magnetic resonances," Science, vol. 317, no. 5834, pp. 83-86, Jul. 2007.

[2] C. Kim and B. Lee, "Analysis of magnetically coupled wireless power transmission for maximum efficiency," Journal of the Korea Electromagnetic En- 
gineering Society, vol. 11, no. 3, pp. 156-160, Sep. 2011.

[3] B. C. Park, J. H. Park, and J. H. Lee, "High-Q spiral zeroth-order resonators for wireless power transmission," Journal of Electromagnetic Engineering and Science, vol. 23, no. 3, pp. 343-354, Sep. 2012.

[4] F. Zhang, S. A. Hackworth, W. Fu, and M. Sun, "The relay effect on wireless power transfer using witricity," in Proceedings of the 14th Biennial IEEE Conference on Electromagnetic Field Computation, Chicago, IL, 2010.

[5] W. Zhong, C. K. Lee, and S. Y. R. Hui, "General analysis on the use of Tesla's resonators in domino forms for wireless power transfer," IEEE Transac-

\section{Ju-Hui Kim}

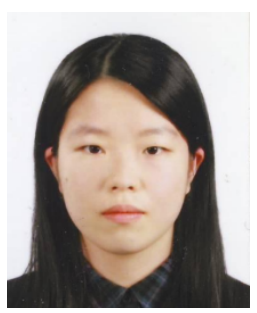

received the B.S. degree in electronic and electrical engineering from Hongik University, Seoul, Korea, in 2012, where she is currently working toward the M.S. degree. Her research interests include wireless power transfer.

\section{Byung-Chul Park}

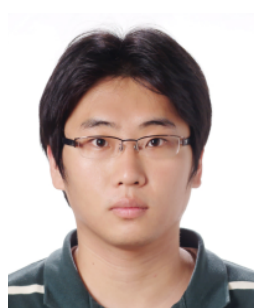

received the B.S. degree in electronic and electrical engineering and the M.S. degree in electronic information and communication engineering from Hongik University, Seoul, Korea, in 2008 and 2010, respectively, where he is currently working toward the Ph.D. degree. His research interests include metamaterial small antenna and wireless power transfer. tions on Industrial Electronics, vol. 60, no. 1, pp. 261-270, Jan. 2013.

[6] D. M. Pozar, Microwave Engineering, 3rd ed., Hoboken, NJ: John Wiley \& Sons, 2005.

[7] J. H. Park, B. C. Park, and J. H. Lee, "Optimum frequency of high Q-factor resonator for magnetic resonance coupling," in Proceedings of the 41st European Microwave Conference, Manchester, UK, 2011, pp. 61-63.

[8] K. Fotopoulou and B. W. Flynn, "Wireless power transfer in loosely coupled links: coil misalignment model," IEEE Transactions on Magnetics, vol. 47, no. 2, pp. 416-430, Feb. 2011.

Jeong-Hae Lee

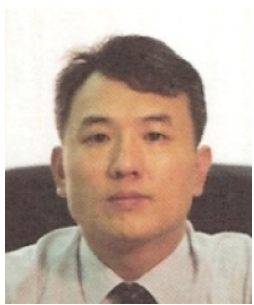

received the B.S. and M.S. degrees in electrical engineering from Seoul National University, Korea, in 1985 and 1988, respectively, and the Ph.D. degree in electrical engineering from the University of California, Los Angeles, in 1996. From 1993 to 1996, he was a Visiting Scientist of General Atomics, San Diego, $\mathrm{CA}$, where his major research was to develop the millimeter wave diagnostic system and to study the plasma wave propagation. Since 1996, he has been at Hongik University, Seoul, Korea, where he is a Professor of Department of Electronic and Electrical Engineering. His current research interests include the microwave/millimeter wave circuits, the millimeter wave diagnostic, the metamaterial RF devices, and wireless power transfer. 